\title{
Structural Phase Transitions and Equations of State for Selenium under Pressures to 129 GPa
}

\author{
T. Krüger and W. B. Holzapfel \\ Fachbereich Physik, Universität-GH-Paderborn, D-4790 Paderborn, Federal Republic of Germany
}

(Received 26 March 1992)

\begin{abstract}
By the use of energy-dispersive $x$-ray diffraction with synchrotron radiation, pressure-induced structural phase transitions and equations of state were studied on Se in the extended pressure range up to $129 \mathrm{GPa}$. In contrast to a previous study on $\mathrm{Se}$ to $50 \mathrm{GPa}$, the higher resolution with synchrotron radiation in the present study allowed for an unambiguous identification of the structure for Se-IV as rhombohedral ( $\beta$-Po type) with one atom in the rhombohedral unit cell, and thus, a close similarity to Te under pressure and Po at higher temperatures is reestablished.
\end{abstract}

PACS numbers: $61.55 . \mathrm{Dc}, 64.30 .+\mathrm{t}, 64.70 . \mathrm{Kb}$

The systematics in the behavior of the elements under high pressures represents an area of increasing activity in recent years due to the advances in diamond-anvil highpressure techniques $[1-3]$ and in theoretical calculations, which reproduce or even predict some of the new highpressure structures and their equations of state (EOS) with remarkable accuracy [4].

Earlier attempts to reveal the structural behavior of Se and $\mathrm{Te}$ in extended pressure regions $[5,6]$ showed rather complex patterns with a total of five different phases for Te below $40 \mathrm{GPa}$ and four phases for Se below $50 \mathrm{GPa}$ with little systematics in the mutual structural sequences.

Since the complex diffraction patterns for Se II, III, and IV were not well resolved with the previous energydispersive $\mathrm{x}$-ray-diffraction (EDXD) technique using a conventional $2-\mathrm{kW} x$-ray generator in the laboratory, higher resolution as well as better collimation and an extended pressure range were provided in the present study by the use of synchrotron radiation (SR) at HASYLAB (Hamburg). The characteristics of this EDXD station as

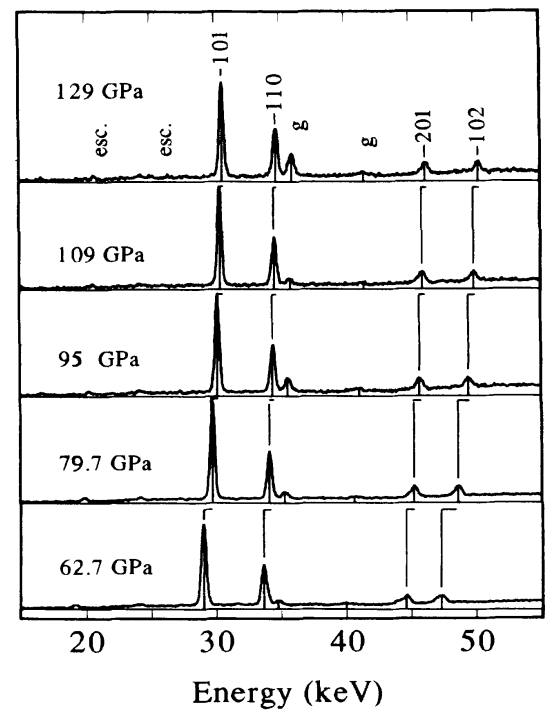

FIG. 1. Energy-dispersive x-ray-diffraction pattern of selenium IV at ambient temperature and different pressures. well as the other experimental details have been described in various previous publications [7-9]. Inconel gaskets with nitrogen as the pressure-transmitting medium were used for the minimization of deviatoric stresses [7]. Pressures were determined with the ruby-luminescence technique [10] and the nonlinear ruby scale [11]. Accordingly, the precision in the pressure evaluation is better than $5 \%$; however, due to the well-known uncertainties in this pressure scale, the accuracy of the given pressure value may be lower than $10 \%$. On the other hand, the $d$ spacings for the nonoverlapping lines shown for instance in Fig. 1 are determined with an accuracy of better than 1 pm, which results in a total uncertainty for the atomic volumes of less than $1 \%$ as shown in Fig. 2.

Typical spectra for Se-IV in the pressure range between 63 and $129 \mathrm{GPa}$ are shown in Fig. 1. The higher resolution and repositioning of the sample with respect to

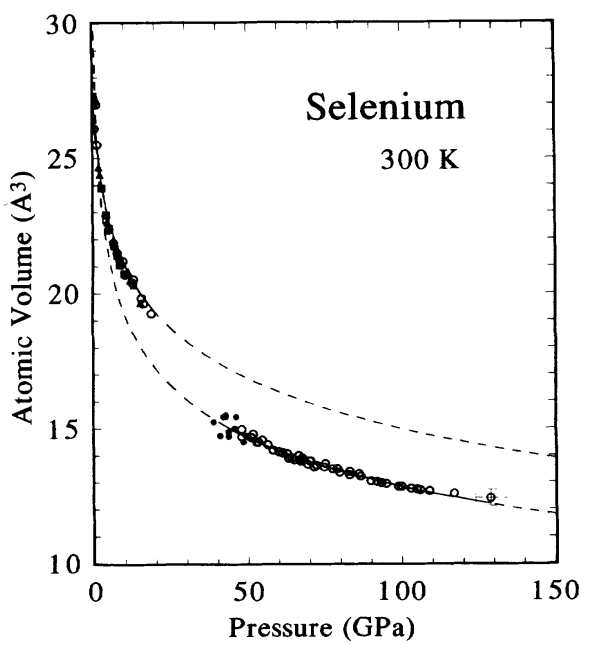

FIG. 2. EOS data at ambient temperature for the trigonal (low-pressure phase) Se-I and the $\beta$-Po-type (high-pressure phase) Se-IV. Present data are marked by open circles. Previous data [6] for Se-IV reevaluated with the present structural assignment are shown by solid circles. Previous data for Se-I are represented by squares and triangles, respectively $[15,6]$. The fitted EOS forms are discussed in the text. 
the well-collimated SR beam allowed for a definite identification of two diffraction lines present also in the previous study as diffraction lines from the gasket (marked by $g$ in Fig. 1). Furthermore, the extended pressure range assured that these lines also showed different pressure shifts and no other lines [except the wellidentified very weak escape peaks (esc.)] were noticeable in the present spectra. Thus, much simpler spectra were recorded for Se-IV. The absence of any lines other than the ones marked with the trigonal indexing of the rhombohedral $\beta$-Po-type structure appears now as definite support for the assignment of this structure type to Se-IV.

As a consequence, a different crystallographic unit cell than previously proposed [6] results from the present data, and, with one atom in this rhombohedral unit cell, a larger atomic volume than previously assigned is found for Se-IV as shown in Fig. 2.

In fact, this new assignment is completely compatible with a reevaluation of the previous data according to the $\beta$-Po-type structure (if one omits the erroneously interpreted escape peaks and gasket lines of the spectra given there) as shown by the solid circles in Fig. 2, which represent these reevaluated data.

For the representation of all EOS data shown in Fig. 2 by smooth curves (solid lines), we use an EOS for strong compression [12,13] represented by the following form:

$$
P=3 K_{0} x^{-5}(1-x) \exp \left\{\left(c_{0}+x c_{2}\right)(1-x)\right\}
$$

where $x=\left(V / V_{0}\right)^{1 / 3}$ scales the (atomic) volume $V$ with respect to $V_{0}$, its value at zero pressure. The three free parameters, the volume $V_{0}$, the bulk modulus $K_{0}$, and its pressure derivative $K_{0}^{\prime}$ at zero pressure, are determined by least-squares refinements of the correlated parameters $V_{0}, K_{0}, c_{0}$, and $c_{2}$ with respect to the experimental data, whereby $c_{2}=\frac{3}{2}\left(K_{0}^{\prime}-3\right)-c_{0}$ and $c_{0}=-\ln \left(3 K_{0} / P_{\mathrm{FG} 0}\right)$ guarantee the correct asymptotic variation at strong compression by the internal scaling with the Fermi gas pressure $P_{\mathrm{FG} 0}=a_{f}\left(Z / V_{0}\right)^{5 / 3}$, which is written here in terms of the universal constant [7] $a_{f}=2336.9 \mathrm{GPa} \AA^{5}$ and the respective electron density $Z / V_{0}$. This refinement results in the following parameters for the trigonal (low-pressure) phase, Se-I,

$$
V_{0}=27.3(2) \AA^{3}, \quad K_{0}=11(2) \mathrm{GPa}, K_{0}^{\prime}=10.5(1),
$$

and for the rhombohedral (high-pressure) phase, Se-IV,

$$
V_{0}=34(1) \AA^{3}, K_{0}=0.76(3) \mathrm{GPa}, K_{0}^{\prime}=12.6(12) \text {. }
$$

Thereby, the values in parentheses represent only the (uncorrelated) statistical errors of these parameters (keeping all other parameters fixed). As discussed previously [14], the possible (correlated) uncertainties in these parameter values are, however, almost an order of magnitude larger, which should be kept in mind, when these (correlated) parameters are compared in future with oth- er experimental or theoretical results. However, these uncertainties do not affect the fitted EOS forms more than shown by the scatter of the data in Fig. 2, and the dashed lines in Fig. 2, representing best (fitted) extrapolations into the mutual regions of instability, now give much narrower boundary conditions to be used in a revision of the structural assignment for the intermediate phases Se-II and Se-III.

Since the spectra for these intermediate phases are, however, still rather complex (with possible phase mixtures) even in the present SR experiments, these assignments represent still some challenging problems.

In any case, with the present (well-supported) assignment of the $\beta$-Po-type structure to Se-IV, a close similarity to $\mathrm{Te}$ is reestablished, since Te-IV has been found [5] to show this same structure. If one now takes into account that the $c / a$ ratio of Te-IV (in hexagonal representation) decreases under pressure and shows a discontinuous change around $28 \mathrm{GPa}$ which results in the bcc structure, Te-V, one can extrapolate also $c / a$ for Se-IV beyond $129 \mathrm{GPa}$ and, by comparison with the critical ratio for the transition from Te-IV to Te- $\mathrm{V}$, one can estimate that a similar transition to a bcc phase, Se-V, should occur at 200(50) GPa, whereby this large uncertainty takes into account that the expected hysteresis may be much larger at these higher pressures.

Thus, the present data point to a close similarity of Se and $\mathrm{Te}$ under pressure. As a result of the simpler structure types observed, leaving only the rhombohedral angle open for optimization, these data should now also stimulate first-principles calculations of EOS and total energies for the group-VI elements under extreme conditions.

This work has been funded by the German Minister for Research and Technology (BMFT) under Contract No. 05440 AXI 4.

[1] A. Jayaraman, Rev. Mod. Phys. 1, 65 (1983).

[2] H. K. Mao, Y. Wu, L. C. Chen, J. F. Shu, and R. J. Hemley, High Pressure Res. 5, 773 (1990).

[3] A. L. Ruoff, H. Xia, H. Luo, and K. Vohra, Rev. Sci. Instrum. 61, 3830 (1990).

[4] D. A. Young, Phase Diagrams of the Elements (University of California, Berkeley, 1991).

[5] G. Parthasarathy and W. B. Holzapfel, Phys. Rev. B 37, 8499 (1988).

[6] G. Parthasarathy and W. B. Holzapfel, Phys. Rev. B 38, 10105 (1988).

[7] W. A. Grosshans, E.-F. Düsing, and W. B. Holzapfel, High Temp. High Pressure 16, 539 (1984).

[8] K. Syassen and W. B. Holzapfel, Europhys. Conf. Abstr. 1A, 75 (1975).

[9] W. B. Holzapfel, in High Pressure Chemistry, edited by H. Kelm (Reidel, Boston, 1978), p. 177. 
[10] R. A. Forman, G. J. Piermarini, J. D. Barnett, and S. Block, Science 176, 284 (1972).

[11] H. K. Mao, P. M. Bell, J. W. Shaner, and D. J. Steinberg, J. Appl. Phys. 49, 3276 (1978).

[12] W. B. Holzapfel, in Molecular Solids under Pressure, edited by R. Pucci and G. Piccitto (North-Holland, Am- sterdam, 1990), p. 61.

[13] W. B. Holzapfel, Europhys. Lett. 16, 67 (1991).

[14] O. Schulte, A. Nikolaenko, and W. B. Holzapfel, High Pressure Res. 6, 169 (1991).

[15] R. Keller, W. B. Holzapfel, and H. Schulz, Phys. Rev. B 16, 4404 (1977). 